\title{
DECISIÓN DE COMPRA DE LA GENERACIÓN Z Y SU RELACIÓN CON EL MARKETING ECOLÓGICO
}

\author{
GENERATION Z PURCHASE DECISION AND ITS RELATIONSHIP WITH \\ ECOLOGICAL MARKETING
}

\author{
Josué Miguel PAZMIÑO RODRÍGUEZ \\ Jorge Manuel CUEVA ESTRADA ${ }^{2}$ \\ Nicolas Armando SUMBA NACIPUCHA ${ }^{3}$ \\ Aurora Nelly LOZANO CASTILLO ${ }^{4}$
}

\begin{tabular}{|lll|}
\hline Recibido & $:$ & 01.05 .2021 \\
Aceptado & $:$ & 08.07 .2021 \\
Publicado & $:$ & 02.08 .2021 \\
\hline
\end{tabular}

\begin{abstract}
RESUMEN: La degradación del ecosistema a nivel global ha preocupado a los diferentes actores de la sociedad y a su vez han emergido nuevas generaciones interesadas en la preservación del medio ambiente. Frente a esta situación las empresas se han visto obligadas a proponer estrategias que preserven por un lado el ecosistema donde desarrollan sus actividades económicas y por otro que busquen captar la atención de los nuevos consumidores preocupados por esta situación. El objetivo que persiguió la investigación fue: Establecer el grado de asociación entre el marketing ecológico (Mkt. Eco.) y el proceso de decisión de compra del consumidor de la generación Z (PDCZ), se realizó un estudio de tipo empírico entre ellas utilizando el coeficiente de correlación Rho de Spearman, la muestra estuvo formada por 687 personas integrantes de la generación Z. El principal resultado obtenido fue la existencia relación lineal entre las variables definida como positiva débil (Rho 0.41 , rho ${ }^{2} 16.81 \%$ ), estos estadísticos demuestran la poca relación existente entre las variables de estudio, conclusión que se justifica dada la recesión económica impuesta por la covid-19, existiendo otros factores como el precio y la calidad que ejercen una mayor incidencia sobre el PDCZ.
\end{abstract}

Palabras clave: Comportamiento del consumidor, decisión de compra, generación z, marketing ecológico, marketing verde.

\begin{abstract}
The degradation of the ecosystem at a global level has concerned different actors in society, and new generations have emerged, interested in preserving the environment. Faced with this situation, companies have been forced to propose strategies that preserve, on the one hand, the ecosystem where they carry out their economic activities and, on the other, capture the attention of the new consumers concerned about that. The purpose of this research was to establish the degree of association between ecological marketing (Mkt. Eco.) and the consumer purchase decision process of Generation Z (PDCZ). An empirical study was carried out by using Spearman's Rho correlation coefficient, and a number of 687 people from Generation $\mathrm{Z}$ represented the sample. The main result obtained was the existence of a linear relationship between the variables defined as weak positive (Rho 0.41 , rho $216.81 \%$ ), which evidenced the little relationship between the study variables, a conclusion that is justified given the economic recession imposed by COVID-19, with other factors such as price and quality that impacted on the consumer purchase decision process of Generation Z.
\end{abstract}

Keywords: Consumer Behavior, COVID-19, Ecological Marketing, Generation Z, Green Marketing, Purchase Decision.

\footnotetext{
${ }^{1}$ Máster en Administración de Empresas. Universidad Politécnica Salesiana. Guayaquil, Ecuador. Email: ipazminor2@est.ups.edu.ec. ORCID: https://orcid.org/0000-0002-3106-319X

${ }^{2}$ Magíster en Administración de Empresas. Universidad Politécnica Salesiana. Guayaquil, Ecuador. Email: jcueva@ups.edu.ec. ORCID: https://orcid.org/0000-0002-3055-1060

3 Máster en Administración de Empresas. Universidad Politécnica Salesiana. Guayaquil, Ecuador. Email: nsumba@ups.edu.ec. ORCID: https://orcid.org/0000-0001-7163-4252

4 Máster en Contabilidad y Auditoría. Universidad Politécnica Salesiana. Guayaquil, Ecuador. Email: alozano@ups.edu.ec. ORCID: https://orcid.org/0000-0002-8554-4052
} 


\section{Journal of the Academy $|63|$}

\section{INTRODUCCIÓN}

El uso indiscriminado de materiales que dañan el medio ambiente es una problemática que siempre ha acompañado a la humanidad y que cada año empeora (Palm y Cueva, 2020). La revolución industrial marcó un punto caótico para la explotación de recursos naturales, debido a que las industrias buscaban aumentar su desarrollo a través del uso de cantidades abismales de materias primas, sin tomar en cuenta los daños adversos que se generarían en el medio ambiente, situación que se ha posicionado como una de las preocupaciones y problemas más importantes que deben resolver las sociedades (Domínguez, 2015).

El ser humano en su intento de satisfacer sus necesidades y deseos ha comprometido el entorno que lo rodea. En su gran mayoría, las materias primas que son utilizadas para diferentes procesos empresariales provienen de recursos no renovables (Rodríguez et al., 2011). En este sentido Deacon (1997) señala que el uso de recursos no renovables sin un respectivo plan de mitigación provoca un deterioro medio ambiental a gran escala. Dado que este tipo de recursos, no poseen la capacidad de regenerarse por sus propios medios (Vargas et al., 2017; Domínguez et al., 2019).

Adicionalmente, se puede mencionar también que el inadecuado manejo de residuos genera un impacto negativo y devastador en el medio ambiente, esto como consecuencia de las diferentes actividades comerciales (Guzmán y Macías, 2012), otra consecuencia de la situación descrita es la aparición de problemas en la salud tanto del ser humano como de las distintas especies que coexisten en el planeta (Escalona, 2014), por lo que se vuelve necesario que los hábitos de producción y consumo tengan un nuevo rumbo, enfocado a la conservación ambiental.

Con todos estos problemas medio ambientales a escala global emergen consumidores o clientes con comportamiento ecológico y con nuevos hábitos de consumo preocupados por ayudar y solucionar estos problemas, en esta línea Left (2002), describe al comportamiento llamado ecológico como pro medioambientales y directamente relacionados con acciones en beneficio del medio ambiente.

Por su parte las firmas deben enfocar sus actividades, pensando en la sostenibilidad, lo que incluye en primer lugar, un adecuado y pertinente proceder ecológico, sin olvidar el contexto económico y social para alcanzar su desarrollo (Gil y Barcellos, 2010). Esto con el fin de 


\section{Journal of the Academy $|64|$}

generar atención y atraer a los consumidores ambientalistas, sin afectar la viabilidad financiera y económica de las industrias (Sánchez, 2016). Para esto las empresas deben hacer uso de análisis de datos más profundos que conlleve a comprender los nuevos gustos y preferencias que los consumidores ambientales y así proponer estrategias pertinentes.

Las organizaciones a través de sus desechos contaminan y destruyen el medio ambiente, las personas muestran interés en la preservación y cuidado del planeta. Frente a esto las empresas buscan estrategias que permitan captar la atención de estos nuevos consumidores preocupados por el medio ambiente. En este contexto surge una nueva vertiente del Marketing - el Mkt. Eco, Salas (2018) lo describe indicando que este nuevo concepto se basa en una comercialización amigable con el medio ambiente y añade que las empresas que logren comercializar sus bienes y servicios mediante este enfoque crearán lazos más fuertes entre sus clientes.

En esta línea, las empresas a nivel global están en búsqueda constante de estrategias que permitan captar la atención de los diferentes tipos de clientes o consumidores (Álvarez y Villacrés 2017), para lo cual las empresas también buscan identificar y caracterizar sus diferentes grupos objetivos, pudiendo ser los millenials un grupo de interés para la mayoría de las organizaciones. En relación con esto, existen varias investigaciones acerca de los Millennials que pretenden comprender su comportamiento de compra, pues se encuentran en edad económicamente productiva, característica que ha resultado de interés para los integrantes del mundo comercial quienes han propuesto estrategias para captar su atención (Cueva-Estrada et al., 2020).

Por otra parte, está emergiendo la Generación Z en el mercado global, pues si bien es cierto aún son muy jóvenes ya que están iniciando o cursando su carrera universitaria (Ortega y Vilanova, 2016; Popescu et al.,2019), también es cierto que en el corto plazo pasarán a ser parte de la fuerza laboral del mercado e independientes económicamente, lo que les permitirá adquirir productos y servicios para cubrir sus necesidades, es por este motivo que la presente investigación plantea analizar la relación entre el marketing ecológico (Mkt. Eco) y el proceso de decisión de compra en las personas que pertenecen a la generación Z (PDCZ), como una estrategia que pudiera ser utilizada por las empresas para captar la atención de este nuevo grupo objetivo 


\section{Journal of the Academy $|65|$}

\section{DESARROLLO}

\section{Marketing ecológico}

El marketing es un conjunto de procesos sociales y administrativos, aplicados con el objetivo de que los integrantes de las sociedades logren satisfacer sus necesidades, preferencias o deseos a través de la obtención de bienes y servicios, para alcanzar esta premisa se vuelve imprescindible conocer claramente el mercado meta de la empresa. (Kotler y Armstrong, 2012). Rodríguez et al., (2020) agregan que se debe abarcar y analizar todas las posibles perspectivas y pensamientos de los consumidores, de tal forma que las organizaciones, los empresarios y los especialistas se adapten a las nuevas tendencias.

Considerando las preferencias de los clientes preocupados por el medio ambiente emerge el Mkt. Eco, también llamado marketing verde, ecomarketing o marketing medioambiental, este tipo de marketing es pertinente tanto para empresas con fines lucrativos y no lucrativos (Esteban et al., 2012; Rugel, et al., 2015).

Lewandowska et al., (2017) señalan que un efecto de aplicar esta vertiente del marketing es la mejora de la imagen corporativa, efecto que permitirá incrementar la rentabilidad de la organización. Añaden que una postura ecológica por parte de la empresa involucra también el compromiso de sus clientes. En este sentido Muñoz (2013) agrega que la participación de la empresa y sus clientes permite que ambos formen parte de la preservación del medio ambiente. Para Mesa (2012) este tipo de marketing inclina los esfuerzos organizacionales hacia estrategias que permitan conservar un aire limpio, agua potable y áreas verdes. Mientras que Calomarde (2000) desde otro enfoque, señala que es la adaptación de las estrategias del marketing comercial migradas al Mkt. Eco, dado que involucra al producto, precio, plaza y promoción; con el objetivo de ayudar a la preservación del ecosistema.

El producto ecológico, se encuentra orientado a la elaboración de bienes y servicios de una manera comprometida con el medio ambiente, utilizando la menor cantidad de recursos, generando la menor cantidad de desperdicios y reutilizando sus componentes, (Calomarde 2000; Chamorro, 2001). Castellano y Urdaneta (2015) agrega que este proceder debe estar presente también dentro de todo el proceso productivo. 


\section{Journal of the Academy $|66|$}

El precio ecológico, otorga el valor monetario según los costos e insumos utilizados en la fabricación del producto o servicio, cabe mencionar lo expuesto por Rivera y Sánchez (2002) quienes indican que el precio de un producto ecológico será mayor al del producto convencional, debiendo ser aceptado por el consumidor o cliente final, esto debido a sus procesos productivos. Y agregan que lo antes expuesto cuestiona el crecimiento del mercado en esta línea de productos. En este sentido Rivera y Brugarolas (2003) señalan que incluso el precio de un producto ecológico puede ser hasta un 50\% superior que uno convencional.

Por otra parte, el punto de venta dentro del Mkt. Eco. busca posicionar productos en el tiempo y lugar adecuado, los cuales permitan satisfacer las necesidades de los clientes. En línea con el Mkt. Eco, el punto de venta también incluye estrategias amigables y responsables con el ambiente, con la finalidad de disminuir el uso de recursos que se emplean para estas actividades. (Chamorro, 2001; Cardona et al., 2018)

Finalmente, la publicidad ecológica tiene como objetivo llegar a los consumidores, a través de un mensaje que comunique las características y beneficios del producto ecológico para el planeta, además de concientizar a su mercado meta, todo esto siempre que la empresa transmita una imagen comprometida con la preservación y cuidado del medio ambiente (Calomarde 2000). Puede agregarse a lo expuesto anteriormente que, para la elaboración de la publicidad ecológica del producto o servicio, toda la escenografía y materiales utilizados deben ser reciclados o que no tengan un impacto negativo para el medio ambiente (Sanmartín et al., 2017).

\section{Generación Z}

No existe un rango de años claramente definido para describir a las personas pertenecientes a la generación Z, Kotler y Armstrong (2012) señalan que la generación del milenio corresponde a las personas nacidas entre los años 1977 y 2000, mientras que Silvestre (2016) señala que los $\mathrm{Z}$ son personas que nacieron a partir del año 2000. Esta generación hace uso constante de tecnología, sus procesos de aprendizaje van ligados a la autoeducación, prefieren lo visual y posee una marcada preocupación por la preservación del medio ambiente, lo cual se ve reflejado en sus hábitos de consumo direccionados a una conciencia ecológica (Pérez-Escoda et al., 2016; Álvarez y Heredia, 2019; Toledo, 2020). 


\section{Journal of the Academy | $67 \mid$}

En la actualidad existen más de 2000 millones de personas que representan a la generación Z, y representan el 32\% de la población mundial (Hernández, 2020). Según la página web del Instituto Ecuatoriano de Estadísticas y Censos (INEC, 2021) la cual posee un contador poblacional actualizable, el Ecuador posee una población de 17602188 personas, si se obtiene el $32 \%$ de esta cifra, la estimación de la cantidad total de integrantes de la Generación Z ecuatorianos sería 5632700 lo que se constituye en la población del presente estudio.

El proceso de decisión de compra según el modelo propuesto por Blackwell et al., (2002) contiene las siguientes etapas: 1) la activación o reconocimiento de la necesidad, 2) búsqueda de información, 3) evaluación de las alternativas, 4) intención o decisión de compra y 5) uso del producto, 6) evaluación del producto, en línea con el proceso descrito Kotler y Armstrong (2012) unen las dos últimas etapas del modelo anterior y la llaman: comportamiento poscompra.

Cabe indicar que durante todo este proceso existen diferentes factores tanto internos como externos al consumidor que pueden incidir sobre la decisión de compra, en este sentido un factor influyente pudiera ser la aplicación de estrategias de Mkt. Eco.

Por lo que a continuación, se plantean las hipótesis que se pretenden comprobar con el desarrollo del estudio:

Hipótesis nula $H_{0}$ : El valor $R h o=0$, No se observa relación entre el marketing de ecológico y el comportamiento de compra del consumidor $\mathrm{Z}$.

Hipótesis alternativa $H_{1}:$ El valor Rho $<>0$, Si se observa relación entre el marketing de ecológico y el comportamiento de compra del consumidor $\mathrm{Z}$.

Se deberá aceptar $H_{0}$ siempre que el valor de Sig. (bilateral) p este por encima del nivel de significancia $\alpha=0.05$, por lo contrario, se deberá rechazar $H_{0}$ siempre que el valor de Sig. (bilateral) $\mathrm{p}$ sea inferior al nivel de significancia $\alpha=0.05$

\section{METODOLOGÍA}

La investigación buscó demostrar la existencia de correlación entre el Mkt. Eco y el PDCZ, para ello se realizó un estudio empírico, el cual permitió la recolección de datos perteneciente 


\section{Journal of the Academy | $68 \mid$}

a cada una de las variables de estudio. Este levantamiento de información se realizó a través de un cuestionario vía online de preguntas dirigido a personas que forman parte de la generación $Z$, con edades entre 18 y 21 años y descartando de la muestra a aquellas personas fuera de este rango de edad. Para las opciones de respuesta se utilizó la escala de Likert del 1 al 5, siendo 1: Nunca, 2: Raramente, 3: Ocasionalmente, 4: Frecuentemente, 5: Muy Frecuentemente. En la tabla 1 se muestran las dimensiones de la variable independiente Mkt. Eco y la variable dependiente PDCZ, la cual se elaboró con información reunida en el estudio de la literatura previa.

\section{Tabla 1}

Operacionalización de las variables

\begin{tabular}{|c|c|c|}
\hline Variable & Dimensión & Indicador \\
\hline Marketing & Producto & 1. Reutilización de materiales. \\
\hline \multirow[t]{6}{*}{ Ecológico } & ecológico & 2. Reducción de materiales. \\
\hline & & 3. Reciclaje de Materiales. \\
\hline & Precio ecológico & 4. Subsidio de un $\%$ del costo de producción. \\
\hline & $\begin{array}{l}\text { Punto de venta } \\
\text { ecológico }\end{array}$ & $\begin{array}{l}\text { 5. Optimización de combustibles no renovables. } \\
\text { 6. Materiales reciclables en el punto de venta. } \\
\text { 7. Almacenamiento amigable con el medio } \\
\text { ambiente. }\end{array}$ \\
\hline & Promoción & 8. Imagen de la empresa. \\
\hline & & 9. Información del producto o servicio. \\
\hline \multirow{8}{*}{$\begin{array}{l}\text { Comportamiento } \\
\text { de compra del } \\
\text { consumidor } \mathrm{Z}\end{array}$} & Factores & 10. Factores económicos \\
\hline & Externos & \\
\hline & Factores Internos & 11. Motivo de compra \\
\hline & & 12. Percepción \\
\hline & & 13. Grupos sociales \\
\hline & Procedo & 14. Reconocimiento de la necesidad \\
\hline & decisión & 15. Búsqueda de información \\
\hline & compra & 16. Evaluación de alternativas \\
\hline
\end{tabular}

Fuente: Elaboración propia con base en Calomarde (2000); Chamorro (2001); Castellano y Urdaneta (2015); Rivera y Sánchez (2002); Cardona et al., (2018); Sanmartín et al. (2017); Blackwell et al. (2002); Kotler y Armstrong (2012) 


\section{Journal of the Academy $|69|$}

El cuestionario desarrollado fue sometido a una prueba de validez realizada por cinco expertos en marketing y ecología, además con el fin de validar la confiabilidad de los resultados obtenidos se aplicó el coeficiente Alfa de Cronbach, cabe indicar que las preguntas se crearon tomando en cuenta las variables de investigación y sus respectivas dimensiones, finalmente con los resultados obtenidos se aplicó un análisis estadístico de correlación utilizando el software SPSS (Statistical Package for Social Sciences).

El tamaño de la muestra del estudio se obtuvo utilizando como población, los datos proporcionados por el INEC (2021) utilizando el 32\% del total de los ecuatorianos es decir 5 632700 , los cuales pertenecen a la generación Z, considerando esta población como infinita. La encuesta fue contestada por 687 personas pertenecientes a la generación $\mathrm{Z}$ con una edad entre 18 y 21 años, constituyéndose en el tamaño muestral empleado en la investigación. Esto implica que el margen de error para el presente estudio fue de $3.74 \%$. En este punto conviene indicar que la muestra seleccionada fue de tipo no probabilístico, dado que los elementos muestreados, contestaron voluntariamente la encuesta vía online.

\section{RESULTADOS}

El resultado de la prueba de fiabilidad Alfa de Cronbach fue: 0.77, esto indica que la confiabilidad de la herramienta de investigación aplicada es aceptable (Hernández et al., 2014). El 58\% de la muestra fueron mujeres, mientras que el $42 \%$ fueron Hombres. En lo referente al nivel de estudio solo el $3 \%$ afirman haber culminado sus estudios universitarios y el $97 \%$ mencionan haber iniciado su carrera profesional. Por otra parte, otro resultado relevante fue que el $68.6 \%$ de los encuestados mencionó al precio como factor determinante en su decisión de compra.

Para evidenciar las hipótesis propuestas en el estudio, se obtuvo primero el total de cada una de las dimensiones tanto de Mkt. Eco., como la del CCCZ. Dado que las distribuciones no son normales ya que se utilizaron variables en el nivel ordinal, se utilizará la prueba no paramétrica coeficiente de correlación de Rho Spearman (Anderson et al., 2008; Hernández et al., 2014) A continuación, la figura 1 muestra una visión general a través de un diagrama de dispersión de la existencia de relación entre las variables. 


\section{Journal of the Academy $|70|$}

\section{Figura 1}

Dispersión Simple del Comportamiento de Compra por Total Mkt. Ecológico

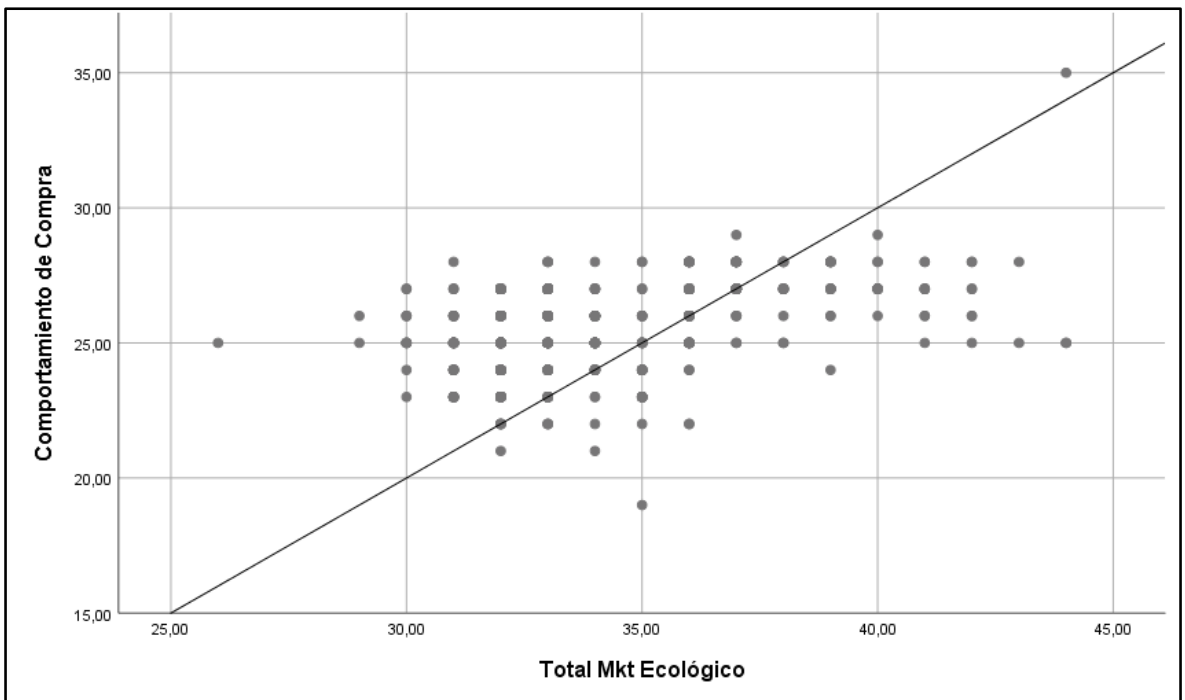

Fuente: Elaboración propia

Ahora se obtendrá el coeficiente de correlación de Rho Spearman, el resultado de este análisis se muestra en la tabla 2.

\section{Tabla 2}

Correlación de Rho de Spearman

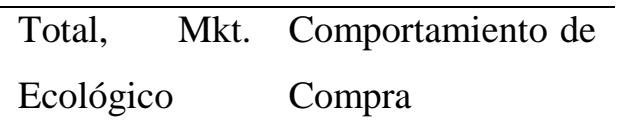

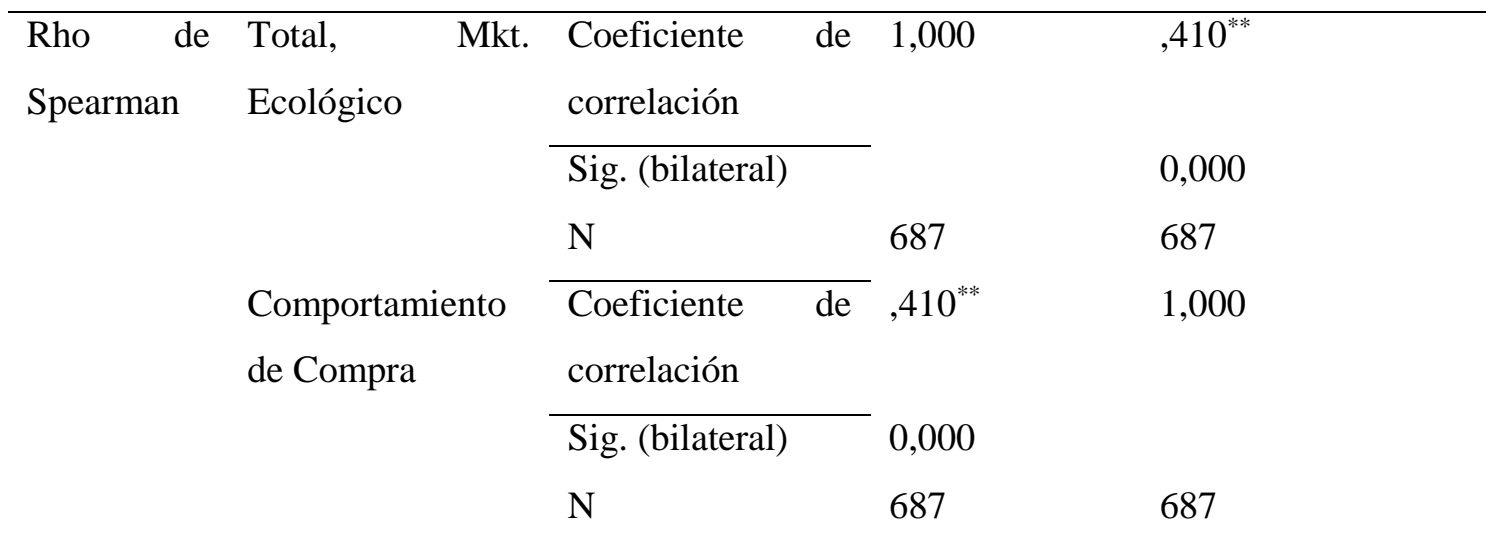

**. La correlación es significativa en el nivel 0,01 (bilateral).

Fuente: Elaboración propia

Dado que el valor de Sig. (bilateral) $\mathrm{p}=0.000$ es menor que nivel de significancia $\alpha=0.05$ la hipótesis nula H0 se debe rechazar y aceptar H1, esto indica la existencia de correlación positiva débil entre las variables con una intensidad según el Rho de Spearman de 0.41. 


\section{Journal of the Academy $|71|$}

(Hernández et al., 2014). El coeficiente de determinación fue de (rho2=0.1681), se puede indicar que la variable Mkt. Eco, explica el 16.81\% del PDCZ, no obstante Creswell (2005) afirma que este porcentaje de coeficiente de determinación no muestra una adecuada predicción de la variable.

De forma más específica la tabla 3 muestra los coeficientes de correlación entre las dimensiones del Mkt. Eco. y el CCCZ.

\section{Tabla 3}

Correlación dimensiones Mkt. Eco. y comportamiento de compra.

\begin{tabular}{|c|c|c|c|c|c|}
\hline & & & Producto & Comportamiento & Intensidad \\
\hline & & & Ecológico & Compra & \\
\hline Rho de & Producto & Coeficiente & 1,000 &,$- 133^{* *}$ & Correlación \\
\hline Spearman & Ecológico & de & & & negativa muy \\
\hline & & correlación & & & débil \\
\hline & & Sig. & & 0,000 & \\
\hline & & (bilateral) & & & \\
\hline & & & Precio & Comportamiento de & \\
\hline & & & Ecológico & Compra & \\
\hline & Precio & Coeficiente & 1,000 &, $146^{* *}$ & Correlación \\
\hline & Ecológico & de & & & positiva muy \\
\hline & & correlación & & & débil \\
\hline & & Sig. & & 0,000 & \\
\hline & & (bilateral) & & & \\
\hline & & & Plaza & Comportamiento de & \\
\hline & & & Ecológico & Compra & \\
\hline & Plaza & Coeficiente & 1,000 &, $264^{* *}$ & Correlación \\
\hline & Ecológico & de & & & positiva débil \\
\hline & & correlación & & & \\
\hline & & Sig. & & 0,000 & \\
\hline & & (bilateral) & & & \\
\hline
\end{tabular}


Journal of the Academy $|72|$

\begin{tabular}{lllll}
\hline & & $\begin{array}{l}\text { Promoción } \\
\text { Ecológico }\end{array}$ & $\begin{array}{l}\text { Comportamiento } \\
\text { Compra }\end{array}$ & de \\
\hline Promoción & Coeficiente & 1,000 &, $614^{* *}$ & $\begin{array}{l}\text { Correlación } \\
\text { positiva } \\
\text { Ecológico }\end{array}$ \\
& de & & & media \\
& correlación & & 0,000 & \\
\cline { 2 - 5 } & Sig. & & & \\
& (bilateral) & & 687 & \\
& $\mathrm{~N}$ & 687 & & \\
\hline
\end{tabular}

**. La correlación es significativa en el nivel 0,01 (bilateral).

Fuente: Elaboración propia

\section{CONCLUSIONES}

Las organizaciones comerciales como uno de los principales actores dentro del planeta han pasado y están obligadas a tener un papel activo y protagónico dentro del cuidado del ecosistema donde desarrollan sus actividades productivas. Esta acción debe abarcar todos los procesos secundarios y esenciales existentes dentro de la organización, es en este punto donde el Mkt. Eco. brinda soporte a las actividades de la empresa. Las acciones de este tipo de marketing abarcan la innovación para el desarrollo de un producto ecológico de tal forma que la empresa cubra los deseos de los consumidores. El precio ecológico incluye, por un lado, asegurar los intereses económicos de la empresa y por otro la aceptación de este por parte del consumidor. El punto de venta ecológico debe minimizar el uso de recursos para su funcionamiento. Por último, la publicidad ecológica debe incluir acciones de concientización para la sociedad.

Las generaciones de personas como parte de la variable de segmentación demográfica son muy utilizadas por las empresas para la selección de sus mercados meta. La generación Z se constituye en uno de esos grupos de interés para las organizaciones pues están entrando a su edad productiva. Cabe indicar que dicha generación posee un pensamiento inclinado y preocupado por la preservación del medio ambiente (Pérez-Escoda et al., 2016; Álvarez y Heredia, 2019; Toledo, 2020).

De forma específica al correlacionar la dimensión promoción ecológica frente al comportamiento de compra del consumidor se obtuvo la existencia de correlación lineal con un coeficiente Rho de Spearman de 0.614 lo que refleja una correlación positiva media. Al 


\section{Journal of the Academy $|73|$}

obtener el coeficiente de determinación 『Rho】^2 se puede indicar que el 37.69\% del PDCZ depende de la promoción o publicidad ecológica realizada por la empresa. Esta situación puede justificarse considerando que este segmento de mercado tiene interés por el cuidado y preservación del planeta.

Al correlacionar de forma integral la variable independiente Mkt. Eco. sobre la variable dependiente CCCZ el resultado del coeficiente Rho de Spearman fue la existencia de correlación lineal con una intensidad de 0.410 lo que indica una correlación positiva débil, dicho de otra forma y utilizando el coeficiente de determinación 『Rho》^2 se puede decir que apenas el 16.81\% del PDCZ depende de las estrategias de Mkt. Eco. generadas por la sociedad comercial. Este resultado puede sostenerse en primer lugar si se considera que la encuesta fue realizada entre los meses de enero y febrero de 2021 época en que el mundo presenta una recesión económica debido a la presencia de la COVID 19, por lo que existirían otros factores que pueden incidir fuertemente sobre la decisión de compra del consumidor de esta generación, en este sentido y según la percepción de los encuestados el $68.6 \%$ reconoció al precio como factor determinante, seguido de la calidad con el $18.5 \%$.

La generación Z se muestra preocupada por el medio ambiente y el cuidado del ecosistema, sin embargo, en su decisión de compra existen factores que ejercen mayor incidencia sobre todo en momentos de recesión económica. Considerando lo expuesto anteriormente los autores invitan al desarrollo de nuevas investigaciones a modo de confirmación de los resultados obtenidos en esta, cuando se recupere la normalidad a nivel global que la COVID 19 ha dejado de lado. Por otra parte, también se recomienda el desarrollo de una investigación donde se construya un modelo a través de regresiones o de ecuaciones estructurales en donde intervengan las variables propuestas en el presente estudio.

Teniendo en cuenta la incidencia de la gestión de talento humano y satisfacción laboral, se puede concluir que la gestión de talento humano cumple un rol interesante en toda empresa para la toma de decisiones dando soluciones factibles, eficaces y eficientes que promuevan satisfacción laboral en la empresa. Ante las proyecciones de los resultados podemos determinar que se logró cumplir con el objetivo general de esta investigación determinando así la relación directa y positiva entre la gestión de talento humano y satisfacción laboral de los empleados de los bancos con mayor capital financiero de Guayaquil. 


\section{REFERENCIAS BIBLIOGRÁFICAS}

Álvarez, R., y Villacrés, F. (2017). La interacción personal y su efecto en la decisión de compra. RETOS. Revista de Ciencias de la Administración y Economía, 7(13), 137-151. https://doi.org/10.17163/ret.n13.2017.05

Álvarez, E., y Heredia, H. (2019). La Generación Z y las Redes Sociales. Una visión desde los $\begin{array}{llll}\text { adolescentes en } & \text { España. }\end{array}$ https://www.revistaespacios.com/a19v40n20/a19v40n20p09.pdf

Anderson, D., Sweeney, D. y Williams, T. (2008). Estadística para administración y economía. $10^{a}$ edición. Cengage Learning.

Blackwell, R. D., Miniard, P. W. y Engel, J. F. (2002). Comportamiento del Consumidor (9 ${ }^{a}$ edición). Editorial Thompson.

Cardona, J., Riaño, D., y Vaca, Y. (2018). Marketing y consumidor green. LuciérnagaComunicación, 12-23. https://dialnet.unirioja.es/descarga/articulo/6563562.pdf

Castellano, S., y Urdaneta, J. (2015). Estrategias de mercado verde utilizadas por empresas a nivel mundial. Estudios interdisciplinarios de ciencias sociales, 17(3), 476-494. https://www.redalyc.org/pdf/993/99342682007.pdf

Calomarde, J. (2000). Marketing ecológico. Pirámide.

Chamorro, A. (2001). El Marketing Ecológico. Medio Ambiente: http://www.5campus.org/leccion/ecomarketing

Creswell, J. W. (2005). Educational research: Planning, conducting, and evaluating quantitative and qualitative research (2a. ed.). Prentice-Hall.

Cueva-Estrada, J.; Sumba-Nacipucha, N. y Villacrés-Beltrán, F. (2020). El marketing de influencias y su efecto en la conducta de compra del consumidor millennial. Revista Suma de negocios, 11 (25), 99-107. https://doi.org/10.14349/sumneg/2020.v11.n25.a1

Deacon, R. (1997). Los Recursos No Renovables y El Medio Ambiente. Revista Española de Economía

Agraria, 179 ,

$1-32$. https://www.mapa.gob.es/app/publicaciones/art_datos_art.asp?articuloid=787\&codrevi $\underline{\text { sta}=R E E A}$

Domínguez, M. (2015). La contaminación ambiental, un tema con compromiso $\begin{array}{lll}\text { social. Producción } & +\quad \text { Limpia, 10(1), }\end{array}$ http://www.scielo.org.co/scielo.php?script=sci_arttext\&pid=S1909$\underline{04552015000100001 \& \operatorname{lng}=\text { en \&tlng }=\text { es }}$

Domínguez, R., León, M., Samaniego, J., y Sunkel, O. (2019). Recursos Naturales, Medio Ambiente y Sostenibilidad. Santiago: Naciones Unidas. https://www.cepal.org/sites/default/files/publication/files/44785/S1900378_es.pdf 
Escalona, E. (2014). Daños a la salud por mala disposición de residuales sólidos y líquidos en Dili, Timor Leste. Revista Cubana de Higiene y Epidemiología, 52(2), 270-277. http://scielo.sld.cu/scielo.php?script=sci_arttext\&pid=S1561$\underline{30032014000200011 \& \operatorname{lng}=\mathrm{es} \& \ln \mathrm{ln}=\mathrm{es}}$

Esteban, Á., Mondéjar, J. y Cordente, M. (2012). Marketing y medio ambiente: Una revisión de la literatura. Impacto ambiental de las actividades económicas (G. Ferrari, J. Montero, J. Mondéjar y M. Vargas, Edits.). Septem Ediciones.

Gil, A., y Barcellos, L. (2010). Los desafíos para la sostenibilidad empresarial en el siglo XXI. $\begin{array}{lllll}\text { Revista Galega de } & \text { Economía, } & 20 & \text { (2), }\end{array}$ https://www2.javerianacali.edu.co/sites/ujc/files/normas_apa_revisada_y_actualizada_ mayo_2019.pdf

Guzmán, M., y Macías, C. (2012). El manejo de los residuos sólidos municipales: un enfoque antropológico. El caso de San Luis Potosí, México. Estudios sociales, 20(39), 235-262. http://www.scielo.org.mx/scielo.php?script=sci_arttext\&pid=S0188$45572012000100009 \& \operatorname{lng}=$ es \&tlng=es.

Hernández, S. (2020). Fortune. Guía para entender a los centennials. https://www.fortuneenespanol.com/leadership/guia-entiendas-centennials/

Hernández, R., Fernández, C y Baptista, M. (2014). Metodología de la investigación Sexta Edición. McGRAW-HILL / INTERAMERICANA EDITORES, S.A.

Instituto Ecuatoriano de Estadísticas y Censos (2021). INEC, Buenas cifras, mejores vidas. Quito, Ecuador. https://www.ecuadorencifras.gob.ec/estadisticas/

Kotler, P., y Armstrong, G. (2012). Marketing. Pearson

Left, E. (2002). Ciencias Sociales y formación medioambiental. Editorial Gedisa.

Lewandowska, A., Witczak, J. y Kurczewski, P. (2017). Green marketing today - a mix of trust, consumer participation and life cycle thinking. Management, 21(2), 28-48. http://dx.doi.org/10.1515/manment-2017-0003

Mesa, M. (2012). Fundamentos de Marketing. Ecoe Ediciones.

Muñoz, V. (2013). Marketing Ecológico. Ediciones Pirámide.

Ortega, I. y Vilanova, N. (2016). Generación Z El último salto generacional. Atrevia, Deusto. http://ethic.es/wp-content/uploads/2016/04/ResumenEjecutivo_GeneracionZ_1403152.pdf

Palm, K. M., y Cueva, J. M. (2020). Incidencia del marketing ecológico de productos alimenticios en la compra del consumidor millennial de Guayaquil. Revista Publicando, 7(25), 11-38. https://revistapublicando.org/revista/index.php/crv/article/view/2079

Pérez-Escoda, A., Castro-Zubizarreta, A., \& Fandos-Igado, M. (2016). Digital skills in the Z generation: Key questions for a curricular introduction in Primary School. [La 


\section{Journal of the Academy $|76|$}

competencia digital de la Generación Z: Claves para su introducción curricular en la Educación Primaria]. Comunicar, 49, 71-79. https://doi.org/10.3916/C49-2016-07.

Popescu, D., Popa, D., y Cotet, B. (2019). Preparando a los estudiantes para la Generación Z: consideraciones sobre el currículo de impresión 3D. Propósitos y Representaciones, 7(2), 240-254. http://www.scielo.org.pe/pdf/pyr/v7n2/a10v7n2.pdf

Rivera, L. y Sánchez, M. (2002). Marketing de productos ecológicos. Colecciones Mediterráneo Económico: "La agricultura mediterránea en el siglo XXI", (2), 159-176. https://www.publicacionescajamar.es/publicacionescajamar/public/pdf/publicacionesperiodicas/mediterraneo-economico/2/2-10.pdf

Rivera, L. y Brugarolas, M. (2003). Estrategias comerciales para los productos ecológicos.

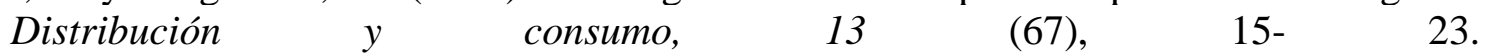
https://www.mapa.gob.es/ministerio/pags/biblioteca/revistas/pdf_DYC/DYC_2003_67 _15_22.pdf

Rodríguez, M., Pineda, D., y Castro, C. (2020). Tendencias de marketing moderno, una revisión teórica. Revista 1 Espacios, https://www.revistaespacios.com/a20v41n27/a20v41n27p26.pdf

Rodríguez, V., Bustamante, L., y Mirabal, M. (2011). La protección del medio ambiente y la salud, un desafío. Revista Cubana de Salud Pública, 37(4), 510-518. https://doi.org/10.1590/s0864-34662011000400015

Rugel, D., Nicolalde, M., y Alava, C. (2015). Plan de Marketing Ecológico para mitigar los impactos ambientales en la Universidad Politécnica Salesiana. RETOS. Revista de Ciencias de la Administración y Economía, 5(9),55-72. https://www.redalyc.org/articulo.oa?id=504550660005

Salas, H. (2018). Marketing ecológico: La creciente preocupación empresarial por la protección del medio ambiente. Fides et Ratio - Revista de Difusión cultural y científica de la Universidad La Salle en Bolivia, 15(15), 151-170. http://www.scielo.org.bo/scielo.php?script=sci_arttext\&pid=S2071$\underline{081 X 2018000100010 \& \operatorname{lng}=\text { es\&tlng=es }}$

Sánchez, N. (2016). La Sostenibilidad en el sector empresarial. Catalunya: UPC. https://upcommons.upc.edu/bitstream/handle/2099.1/18820/TFM_NSanchez_La\%20so stenibilidad\%20en\%20el\%20sector\%20empresarial.pdf

Sanmartín R., Gladis S., Zhigue, R., y Alaña, T. (2017). EL RECICLAJE: UN NICHO DE INNOVACIÓN Y EMPRENDIMIENTO CON ENFOQUE AMBIENTALISTA. $\begin{array}{lllll}\text { Revista Universidad } \quad y \quad \text { Sociedad, } & 9(1), & 36-40 .\end{array}$ http://scielo.sld.cu/scielo.php?script=sci_arttext\&pid=S2218$\underline{36202017000100005 \& \operatorname{lng}=e s \& t \operatorname{lng}=e s \text { Silvestre }}$

Silvestre, E. (2016). CONOCIENDO LA PRÓXIMA GENERACIÓN DE ESTUDIANTES UNIVERSITARIOS DOMINICANOS A TRAVÉS DE LAS REDES SOCIALES. Red de revistas científicas de América latina, el caribe, España y Portugal, 41(3) 475-503. https://www.redalyc.org/pdf/870/87048049003.pdf 
Toledo, V. (2020). Generación Z el Fin Del Mundo Tal Y Como Lo Conocemos. Universidad de Navarra.

Vargas, N., Bustos, C., Ordoñez, O., Calle, M., y Noblecilla, M. (2017). Uso y aprovechamiento de los recursos naturales y su incidencia en el desarrollo turístico local sostenible. Caso Pasaje. Revista interamericana de ambiente y turismo, 13(2), 206-217. https://dx.doi.org/10.4067/S0718-235X2017000200206 